\title{
A VARIATIONAL MODEL IN IMAGE PROCESSING WITH FOCAL POINTS
}

\author{
Andrea Braides ${ }^{1}$ And Giuseppe RieY ${ }^{2}$
}

\begin{abstract}
We propose a model for segmentation problems involving an energy concentrated on the vertices of an unknown polyhedral set, where the contours of the images to be recovered have preferred directions and focal points. We prove that such an energy is obtained as a $\Gamma$-limit of functionals defined on sets with smooth boundary that involve curvature terms of the boundary. The minimizers of the limit functional are polygons with edges either parallel to some prescribed directions or pointing to some fixed points, that can also be taken as unknown of the problem.
\end{abstract}

Mathematics Subject Classification. 68U10, 94A08, 49J45.

Received June 6, 2007. Revised January 12, 2008.

Published online July 4, 2008.

\section{INTRODUCTION}

Within the theories of Image Processing, variational methods are frequently used, in particular to ensure existence and approximability for segmentation problems, which consist in recovering some image contours from a given input $[4,8,21,23,29]$. The mathematical approach for such problems can be summarized as follows: given an input image $g \in L^{\infty}(\Omega)$ (where $\Omega$ denotes the image domain) solve a minimum problem of the form:

$$
\min \left\{\alpha \int_{\Omega}|u(x)-g(x)|^{2} \mathrm{~d} x+E(u, K)\right\}
$$

for a suitable energy $E$, where $K \subset \Omega$ is union of $C^{1}$-curves and $u$ ranges over a suitable space of functions defined in $\Omega \backslash K$. The solution $(u, K)$ of (1.1) is a pair where $K$ represents the relevant contours of the image and $u$ is a regularization of $g$. The first term $\int_{\Omega}|u(x)-g(x)|^{2} \mathrm{~d} x$ is a fidelity term to the input image, while the second one $E(u, K)$ can be seen as an energy which penalizes large variations of $u$ and forces the gradient of $u$ to not oscillate too much outside $K$ (typically there are large oscillations of $\nabla u$ when noise is present in the image) while at the same it penalizes over-segmentation; $\alpha$ is a parameter which balances the two terms. One of the first such functionals has been introduced by Mumford and Shah [24-26] taking $E$ of the following form:

$$
E(u, K)=\beta_{1} \int_{\Omega \backslash K}|\nabla u(x)|^{2} \mathrm{~d} x+\beta_{2} \mathcal{H}^{1}(K),
$$

\footnotetext{
Keywords and phrases. $\Gamma$-convergence, curvature functionals, segmentation problems, image processing.

${ }^{1}$ Dipartimento di Matematica, Università di Roma 'Tor Vergata', Via della Ricerca scientifica 1, 00133 Roma, Italy. riey@mat.unical.it

2 Dipartimento di Matematica, Università della Calabria, Via P. Bucci, 87036 Arcavacata di Rende (CS), Italy.
} 
where $\mathcal{H}^{1}(K)$ denotes the one-dimensional Hausdorff measure of the set $K$. Within the Calculus of Variations (1.1) is called a free-discontinuity problem and, denoted by $F$ the functional $F(u, K)=\alpha \int_{\Omega} \mid u(x)-$ $\left.g(x)\right|^{2} \mathrm{~d} x+E(u, K)$ and by $\mathcal{C}$ the family of all the subsets of $\mathbb{R}^{2}$ which are union of $C^{1}$-curves, problem (1.1) becomes:

$$
\min \left\{F(u, K): u \in W^{1,2}(\Omega \backslash K), K \in \mathcal{C}\right\},
$$

where $W^{1,2}(\Omega \backslash K)$ is the usual Sobolev space. To obtain existence, uniqueness, regularity and approximations of solutions for this problem, it is convenient to rewrite (1.1) in a weaker form, as

$$
\min _{u \in S B V(\Omega)}\left\{\alpha \int_{\Omega}|u(x)-g(x)|^{2} \mathrm{~d} x+\mathcal{E}(u)\right\}
$$

where $\mathcal{E}(u)=\beta_{1} \int_{\Omega \backslash S_{u}}|\nabla u(x)|^{2} \mathrm{~d} x+\beta_{2} \mathcal{H}^{1}\left(S_{u}\right)$ and $S B V(\Omega)$ denotes the space of special functions with bounded variations on $\Omega$ introduced by De Giorgi and Ambrosio (see [3,10,19]). A key point for the application of the direct method of the Calculus of Variations is the lower semicontinuity of the functional $\mathcal{E}$ with respect to the $L^{1}$-convergence, with respect to which the problems in (1.4) are coercive.

From the numerical point of view, the computation of solutions of (1.4) is rather difficult due to the presence of the term $\mathcal{H}^{1}\left(S_{u}\right)$, and a central issue is the approximation of energies such as (1.2) by more handy energies [10]. An underlying feature of such approximation is that the jump set $S_{u}$ is 'blurred' so that it becomes a two-dimensional set, to which various approximation procedures can be applied. In a recent paper by Braides et al. [13] it is shown how we can approximate $\mathcal{E}$ by functionals defined on pairs function-set

$$
\widetilde{\mathcal{E}}_{\varepsilon}(u, A)=\beta_{1} \int_{\Omega}|\nabla u(x)|^{2} \mathrm{~d} x+\frac{1}{2} \beta_{2} \mathcal{H}^{1}(\partial A), \quad|A| \leq \varepsilon ;
$$

the introduction of the perimeter functional $\mathcal{H}^{1}(\partial A)$ allows then to use 'classical' approximation results as in the seminal paper by Modica and Mortola [22]. This is a formalization of an argument that is present for example in the classical approximation by elliptic functionals by Ambrosio and Tortorelli [2] or in the finite-element schemes by Chambolle and Dal Maso [14-16], that actually is immediately derived from [13].

In this paper we investigate the possibility of adding to the procedure above the constraint that the jump set $S_{u}$ be composed of curves pointing in some fixed directions. We have in mind the practical problem of image reconstruction in an urban environment, where the relevant contours either are parallel to some fixed directions (as for edges of buildings, walls, etc.) or point towards some 'focal point' (as for street pavements, etc.). A typical application would be segmenting aerial images of cities in order to find buildings, which often have the sides aligned with each other. The idea to develop the proposed variational model derives from the complexity of a parametric model representing polygonal boundaries, with the corresponding difficulties deriving from topological constraints and from the high computational cost of combinatoric models. Even tough our paper has a 'modeling' character and no numerical results are presented, potential applications to Numerical Analysis should be kept in mind. The energies we propose identify corner points as concentration points of curvature energies and, although more complex from the theoretical and numerical standpoint, may provide an easier solution, in that they identify at the same time the corner points and the segmentation. While some years back the treatment of curvature functionals would have seemed computationally unreasonable, it now seems a feasible alternative by the use of suitable diffuse interface approximations.

We will concentrate on the case corresponding to the 'perimeter functional'; i.e., when the unknown $u$ in (1.3) is a characteristic function, or, equivalently the unknown is a set $A$ and the functional $\mathcal{E}$ reduces to $\beta_{2} \mathcal{H}^{1}(\partial A)$. In this case the simplest constraint that we may add is prescribing the possible directions of the edges of the unknown set $A$. Unfortunately, this constraint is not closed under $L^{1}$ convergence. It may be seen [1] that minimizing sequences may develop increasingly wiggly boundary, and converge to a solution of a problem where the constraint is lost and the usual perimeter is substituted by a different anisotropic perimeter. This over-segmentation of the boundary of $A$ can be avoided by introducing an additional term accounting for 
the cardinality of the set $\widehat{A}$ of end-points of the segments composing $\partial A$. In this direction, a simple functional is

$$
\beta_{2} \mathcal{H}^{1}(\partial A)+\beta_{3} \sharp(\widehat{A})
$$

with the constraint that the tangent $\tau$ to $\partial A$ belongs to a fixed finite set of directions. Clearly, the introduction of the second term greatly simplifies the problem from the viewpoint of the Calculus of Variations, as it provides a bound on the number of segments composing $\partial A$, but it also complicates numerical issues as it introduces a zero-dimensional unknown set.

The functional in (1.5) (but without the constraints on $\nu$ ) can also be seen as a particular form of functionals $E(u, K)$ taking into account the curvature of the unknown contour $K$ of the following form:

$$
E(u, K)=\beta_{1} \int_{\Omega \backslash K}|\nabla u|^{2} \mathrm{~d} x+\beta_{2} \mathcal{H}^{1}(K)+\beta_{3} \sharp(\widehat{K})+\beta_{4} \int_{K} \kappa^{2}(x) \mathrm{d} \mathcal{H}^{1}(x),
$$

where $\kappa(x)$ denotes the curvature of $K$ (which is supposed to be a finite union of $C^{2}$-curves) in a generic point $x, \widehat{K}$ is the set of endpoints of curves of $K$ and $\sharp(\widehat{K})$ is the number of points in $\widehat{K}$. Analysis of properties of functionals involving curvature terms can be found for instance in $[5,6,17,20]$. We refer to [26] for a description of applications to image processing of such a kind of functionals, which are useful for instance to reconstruct overlapping shapes and in particular to recover the edges of regions with occlusion. If we take $\beta_{4}=+\infty$ the curvature term is replaced by the constraint that the competitors $K$ in (1.1) (with $E(u, K)$ given by (1.6)) be union of segments. It is usual to refer to this kind of problem as an edge-detection problem. The approximation of such problems is a much harder issue than for the Mumford-Shah functional and has been addressed by Braides and March [12].

In the present paper we focus our attention on edge-detection functionals depending only on characteristic functions $u=\chi_{A}$, so that $K=\partial A$ as in (1.5), and with the constraint that the contours be parallel to some preferred directions or pointing to some focal points. Mathematically speaking, this can be phrased as follows: let $\nu_{1}, \ldots, \nu_{N} \in S^{1}$ be some fixed directions and let $p_{1}, \ldots, p_{M} \in \mathbb{R}^{2}$ be some fixed points, and define $D_{x}:=\left\{\nu_{i}\right\}_{i=1, \ldots, N} \cup\left\{x-p_{j}\right\}_{j=1, \ldots, M}$; we want to solve (1.1) with $E(u, K)=E(K)$ given by (1.5) under the additional constraint that, if $\tau(x)$ is the tangent vector at a point $x$ of a curve of $K$, then $\tau(x)$ must be parallel to an element of $D_{x}$. It is interesting to note that in these kinds of problems the directions $\nu_{i}$ and the points $p_{i}$ can also be considered as an unknown, and a further minimization is possible to determine their optimal choices. Again, this functional is numerically hard to treat due to the term $\sharp(\widehat{K})$.

Scope of this paper is to present a class of functionals of the type above defined as limits of curvature functionals defined on boundary of sets, the underlying idea being that the counting functional can be considered as a degenerate curvature functional. With this approximation the zero-dimensional unknown set $\widehat{K}$ is substituted by a one-dimensional unknown set, to which approximation arguments by elliptic functionals in the spirit of the Modica and Mortola result can be applied (see [11,22]). We prove a result of $\Gamma$-convergence which generalizes that given by Braides and Malchiodi in [11], where the case without focal points is considered. We refer to [11] and the references quoted there also for other possible applications (for instance elastic crystals) of such a kind of functionals.

In the sequel for simplicity we first assume $\Omega$ to be equal to the whole plane $\mathbb{R}^{2}$ and in a later section we show how it is possible to generalize the results to a bounded domain. Let $\varphi: \mathbb{R}^{2} \times S^{1} \rightarrow \mathbb{R}$ be a function such that $\varphi(x, \cdot)$ vanishes only on $D_{x}$ and, given a function $g: \mathbb{R}^{2} \rightarrow \mathbb{R}$ and $\varepsilon>0$, consider the family of minimum problems

$$
\min \left\{\int_{\partial A}\left(\frac{\varphi(x, \tau(x))}{\varepsilon}+\varepsilon \kappa^{2}(x)\right) \mathrm{d} \mathcal{H}^{1}(x)+\int_{\mathbb{R}^{2}}\left|\chi_{A}-g\right|^{2} \mathrm{~d} x\right\}
$$

where the minimum is taken among subsets of $\mathbb{R}^{2}$ with $C^{2}$-boundary and $\kappa(x)$ and $\tau(x)$ denote respectively the curvature and the unit vector of $\partial A$ at $x ; \chi_{A}$ is the characteristic function of the set $A$. We want to rigorously characterize the properties of the limit of minimizers under the assumption of equi-boundedness on their perimeters, showing that they also solve a minimum problem. To compute the form of the limit energy 
with respect to the $L^{1}$-convergence (of characteristic functions), we use the tools of $\Gamma$-convergence. Since the second term in (1.7) is seen to be a $L^{1}$-continuous perturbation, it is enough to prove the $\Gamma$-convergence of the first term. Therefore we focus our attention on:

$$
G_{\varepsilon}(E):=\int_{\partial E}\left(\frac{\varphi(x, \tau(x))}{\varepsilon}+\varepsilon \kappa^{2}(x)\right) \mathrm{d} \mathcal{H}^{1}(x)
$$

Loosely speaking we expect that for $\varepsilon$ small the minimizers have the sides either parallel to the directions $\nu_{i}$ or pointing to the focal points. In particular we prove that the minimizers, up to subsequence, converge to the solutions of a minimum problem for a functional defined on a suitable class of polygons. For a polygon $P$ with Lipschitz boundary (that means at each vertex only two segments meet) and with a finite number of vertices (we shall call "regular" this kind of polygons), the limit functional has the following form:

$$
\mathcal{G}(P)=\sum_{v \in V(P)} g\left(p, v^{+}(p), v^{-}(p)\right)
$$

where $V(P)$ denotes the set of all vertices of $P, v^{-}(p), v^{+}(p)$ are the direction of the two segments meeting at $p$ and $g$ is a continuous function such that $g\left(\cdot, v_{1}, v_{2}\right)$ vanishes in $\left\{p_{j}\right\}_{j=1, \ldots, M}$ (explicitly computed from $\varphi$, see (3.3)). On general $P$ polygons, the functional is defined on $P$ as the lower semi-continuous extension of $G$ :

$$
G(P)=\inf \left\{\liminf _{n \rightarrow+\infty} \mathcal{G}\left(P_{n}\right): P_{n} \rightarrow P, P_{n} \text { regular }\right\} .
$$

Differently from the problem studied in [11], since $g\left(\cdot, v_{1}, v_{2}\right)$ tends to 0 as $p$ goes to some $p_{j}$ (in particular we have that $\left.g\left(p, v_{1}, v_{2}\right)=O\left(\left|p-p_{j}\right|\right)\right)$, this functional can be finite also on polygons with a countable set of vertices accumulating at focal points. Moreover, as in [11], the problem is complicated by the usual non-local effects that are characteristic of curvature energies (see also $[6,7]$ ).

For the numerical applications we have in mind, functionals $G_{\varepsilon}$ have themselves to be approximated by a diffuse interface in the spirit of Modica and Mortola. For a discussion of different possibilities for this approximation, which do not bear new difficulties, we refer to [12], where also it is highlighted how the use of a (simpler version of a) long-standing conjecture by De Giorgi recently proved by Röger and Schätzle [27] may contribute to simplifying these functionals.

Note that we make an explicit choice of $\varphi$ as a quadratic function of $\nu_{i}$ and $p_{j}$ (see (3.1)) so that a possible interpretation of $\left\{\nu_{i}\right\}$ and $\left\{p_{j}\right\}$ as unknowns could be easily implemented. This choice has the drawback of an uneven treatment of corners. General $\varphi$ can also be considered, but this inconvenience cannot be completely overcome (except for trivial cases when we only have two directions; see [11] and the Appendix of [9]).

\section{Notation AND DEFINITIONS}

We denote by $\mathcal{A}$ the family of all the open sets of $\mathbb{R}^{2}$ with $C^{2}$ boundary. For $\delta>0$ and $q \in \mathbb{R}^{2}$ we denote by $B_{\delta}(q)$ the open ball centered in $q$ and of radius $\delta$. Where there is no risk of confusion we identify $S^{1}$ with $\mathbb{R}$ modulo $2 \pi$. If $\nu_{1}$ and $\nu_{2}$ belong to $S^{1}$, we denote by $L\left(\nu_{1}, \nu_{2}\right)$ the length of the shortest arc (in $S^{1}$ ) linking $\nu_{1}$ and $\nu_{2}$. Set $J:=\left(\begin{array}{cc}0 & -1 \\ 1 & 0\end{array}\right)$ and for $v \in \mathbb{R}^{2}$ define $v^{\perp}:=J v$.

For $a, b \in \mathbb{R}$, let $\gamma:[a, b] \rightarrow \mathbb{R}^{2}$ be the parameterization of a piecewise- $C^{1}$ curve $C$. (When there is no risk of confusion we use the term "curve" indifferently for the support $C$ and the parameterization $\gamma$.) If $x=\gamma(t) \in C$ is such that $\gamma$ is differentiable in $t$ and $\dot{\gamma}(t) \neq 0$, then we say that $x$ is a regular point of $C$ and we denote by $\tau(x):=\frac{\dot{\gamma}(t)}{|\dot{\gamma}(t)|}$ and $\nu(x):=\tau(x)^{\perp}$ the unit tangent and normal vectors at $x$ respectively. We use the variable $s$ when we parameterize $C$ by means of the arc length, that is when $|\dot{\gamma}(s)|=1$ for all $s \in[0, L(C)]$ (here $L(C)$ is the length of $C$ ). If $x$ is a point where $\gamma$ is not $C^{1}$ then we call $x$ a vertex of $C$. We denote by $V(C)$ the set 
of all vertices of $C$. If $C$ is $C^{2}$ in $x$, we denote by $\kappa(x)$ the curvature of $C$ at $x$. Recall that, if $x=\gamma(s)$ (and $s$ is the arc length), then $\kappa(x)=\left|\gamma^{\prime \prime}(s)\right|$.

If $\gamma^{1}:\left[a_{1}, b_{1}\right] \rightarrow \mathbb{R}^{2}$ and $\gamma^{2}:\left[a_{2}, b_{2}\right] \rightarrow \mathbb{R}^{2}$ are such that $\gamma^{1}\left(b_{1}\right)=\gamma^{2}\left(a_{2}\right)$ then we denote by $\gamma^{1} * \gamma^{2}$ the curve defined on $\left[a_{1}, b_{1}+b_{2}-a_{2}\right]$ and parameterized by

$$
\gamma^{1} * \gamma^{2}(t)=\left\{\begin{array}{ll}
\gamma^{1}(t) & t \in\left[a_{1}, b_{1}\right] \\
\gamma^{2}\left(t-b_{1}+a_{2}\right) & t \in\left[b_{1}, b_{1}+b_{2}-a_{2}\right]
\end{array} .\right.
$$

We say that $C$ is a closed curve if $\gamma(a)=\gamma(b)$.

Given a sequence of curves $C_{n}$, we say that $C_{n}$ converge uniformly if their parameterizations are defined on the same interval $[a, b]$ and $\left\{\gamma_{n}\right\}$ converge uniformly in the usual sense.

\section{1. $\Gamma$-convergence}

In order to describe the convergence of minimum problems we will use the notion of $\Gamma$-convergence $[10,18]$. We recall its definition and the main properties we will use.

Definition 2.1. Let $X$ be a topological space and let $F_{n}, F: X \rightarrow \mathbb{R} \cup\{+\infty\}$. Define the $\Gamma$-liminf and the $\Gamma-\lim \sup$ of $F_{n}$ as:

$$
\begin{aligned}
& \Gamma-\liminf _{n \rightarrow+\infty} F_{n}(x):=\inf \left\{\liminf _{n \rightarrow+\infty} F_{n}\left(x_{n}\right): x_{n} \rightarrow x\right\} \\
& \Gamma-\limsup _{n \rightarrow+\infty} F_{n}(x):=\inf \left\{\limsup _{n \rightarrow+\infty} F_{n}\left(x_{n}\right): x_{n} \rightarrow x\right\} .
\end{aligned}
$$

We say that $F_{n} \Gamma$-converges to $F$ if for all $x \in X$ we have:

$$
\Gamma-\liminf _{n \rightarrow+\infty} F_{n}(x)=\Gamma-\limsup _{n \rightarrow+\infty} F_{n}(x)=F(x) .
$$

We say that the family of functionals $\left\{F_{\varepsilon}\right\}_{\varepsilon \in \mathbb{R}} \Gamma$-converges to $F$ as $\varepsilon \rightarrow 0$ if $\left\{F_{\varepsilon_{h}}\right\} \Gamma$-converges to $F$ for every subsequence $\left\{\varepsilon_{h}\right\}$ converging to zero as $h \rightarrow+\infty$.

Remark 2.2. It is easy to show that (2.1) is equivalent to require that for all $x \in X$ the two following conditions hold:

- ( $\Gamma$-liminf inequality) $F(x) \leq \liminf _{n \rightarrow+\infty} F_{n}\left(x_{n}\right)$ for every sequence $\left\{x_{n}\right\}$ converging to $x$;

- ( $\Gamma$-lim sup inequality) there exists a sequence $\left\{x_{n}\right\}$ converging to $x$ such that $F(x) \geq \limsup _{n \rightarrow+\infty} F_{n}\left(x_{n}\right)$.

Definition 2.3. A sequence of functionals $F_{n}: X \rightarrow \mathbb{R} \cup\{+\infty\}$ is said to be equicoercive if for any sequence $\left\{x_{n}\right\}$ such that $\sup _{n} F_{n}\left(x_{n}\right)<+\infty$ there exists a convergent subsequence.

Theorem 2.4. Let $\left\{F_{n}\right\}$ be a sequence of equicoercive functionals defined on $X$ and $\Gamma$-converging to $F$. Then there exists $\min _{X} F$ and $\min _{X} F=\lim _{n \rightarrow \infty} \inf _{X} F_{n}$. Moreover, if $x_{n}$ is a minimizer of $F_{n}$, then every limit of a subsequence $\left\{\begin{array}{l}X \\ x_{n_{k}}\end{array}\right\}$ of $\left\{x_{n}\right\}$ is a minimizer of $F$.

Proposition 2.5. Let $\left\{F_{n}\right\}$ be a sequence of functionals $\Gamma$-converging to $F$ and let $G$ be a continuous functional. Then the sequence $\left\{F_{n}+G\right\} \Gamma$-converges to $F+G$.

\subsection{Admissible polygons}

Let $N \geq 0$ and $M \geq 0$ be such that $N+M \geq 2$; in the whole paper $\tau_{1}, \ldots, \tau_{N}$ are fixed vectors belonging to the unit circle $S^{1}$ (we shall call "directions" such a kind of vectors) and $p_{1}, \ldots, p_{M}$ are fixed points in $\mathbb{R}^{2}$ (in the paper we call them focal points). For each $x \in \mathbb{R}^{2}$ we set:

$$
D_{x}:=\left\{\nu_{i}\right\}_{i=1, \ldots, N} \cup\left\{x-p_{j}\right\}_{j=1, \ldots, M} .
$$



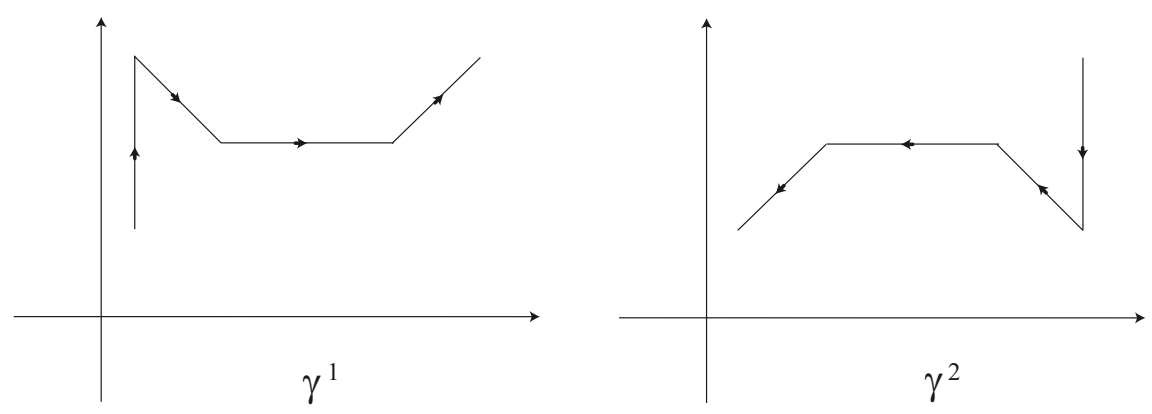

Figure 1. Here are pictured the two paths $\gamma^{1}$ and $\gamma^{2}$.

Definition 2.6 (admissible and regular polygons). We define $\mathcal{P}$ as the class of all the polygons $P$ such that, for almost every $x \in \partial P$, there exists $c \in \mathbb{R}$ such that $c \tau(x)$ belongs to $D_{x}$ (we call admissible such a polygon). We say that $P \in \mathcal{P}$ is regular if (it is admissible and) $V(P)$ is finite and $\partial P$ is Lipschitz (in other words an admissible polygon is regular if at each vertex of its boundary only two segments meet). We denote by $\mathcal{R}$ the class of all the regular admissible polygons.

Definition 2.7. For $P \in \mathcal{P}$ and $v \in V(\partial P)$ let $n$ be the number of the segments which meet at $v$. (Note that $n$ is an even number.) We define the multiplicity of $v$ as $\frac{n}{2}$.

Remark 2.8. If $P$ belongs to $\mathcal{R}$, then each vertex of $P$ has multiplicity 1.

For $P \in \mathcal{P}$ we define a side of $P$ to be the closure of a component of $\partial P \backslash V(P)$. If $q_{1}, q_{2} \in \mathbb{R}^{2}$, we set:

$$
\left[q_{1}, q_{2}\right]:=\left\{z \in \mathbb{R}^{2}: z=q_{1}+t\left(q_{2}-q_{1}\right), t \in[0,1]\right\} \quad \text { and } \quad\left(q_{1}, q_{2}\right):=\left\{z \in \mathbb{R}^{2}: z=q_{1}+t\left(q_{2}-q_{1}\right), t \in(0,1)\right\} \text {. }
$$

Given $q_{1}, q_{2}, q_{3}, q_{4} \in \mathbb{R}^{2}$, we say that the segments $\left[q_{1}, q_{2}\right]$ and $\left[q_{3}, q_{4}\right]$ do not intersect transversally if $\left(q_{1}, q_{2}\right) \cap$ $\left(q_{3}, q_{4}\right)=\emptyset$.

Define $\Upsilon=\left\{\gamma^{1}, \ldots, \gamma^{k}\right\}$ as an element of the class

$$
\mathcal{C}:=\left\{\begin{array}{ll}
\left\{\gamma^{1}, \ldots, \gamma^{k}\right\} \text { such that for all } i=1, \ldots, k: & \frac{\gamma^{i}:\left[a_{i}, b_{i}\right] \rightarrow \mathbb{R}^{2} \text { is Lipschitz }}{\left|\dot{\gamma}^{i}(t)\right|} \in D_{\gamma(t)} \text { a.e. } t \in[0,1] \\
& \gamma^{i}\left(b_{i}\right)=\gamma^{i+1}\left(a_{i+1}\right)
\end{array}\right\} .
$$

For $\Upsilon \in \mathcal{C}$ define the image $\operatorname{Im}(\Upsilon)$ as the image of the path $\gamma^{1} * \ldots * \gamma^{k}$ and the support $S(\Upsilon)$ as the subset of $\operatorname{Im}(\Upsilon)$, where a segment, if it is run an equal number of times in opposite ways, is erased. An example of an element of $\mathcal{C}$ (for which the image and the support are different) is shown below: if we consider the two curves $\gamma^{1}$ and $\gamma^{2}$ in Figure $1, \operatorname{Im}(\Upsilon)$ and $S(\Upsilon)$ are drawn in Figure 2.

We define $\tilde{\mathcal{C}}$ as the class of finite unions of elements of $\mathcal{C}$ and, if $\Upsilon^{1}, \ldots, \Upsilon^{m}$ belong to $\mathcal{C}$, we write: $\Upsilon=$ $\left[\Upsilon^{1}, \ldots, \Upsilon^{m}\right] \in \tilde{\mathcal{C}}$. For $\Upsilon \in \tilde{\mathcal{C}}$ we set:

$$
\operatorname{Im}(\Upsilon):=\bigcup_{\alpha=1}^{m} \operatorname{Im}\left(\Upsilon^{\alpha}\right) \text { and } S(\Upsilon):=\bigcup_{\alpha=1}^{m} S\left(\Upsilon^{\alpha}\right)
$$

We say that $\Upsilon^{\alpha}$ is a component of $\Upsilon$.

Remark 2.9. It is important not to confuse the components of $\Upsilon$ with the connected components of $S(\Upsilon)$. 


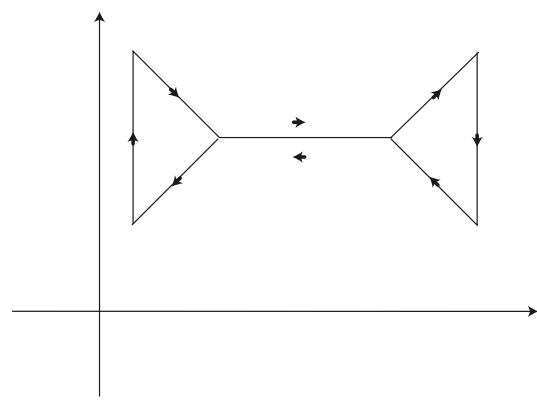

$\operatorname{Im}\left(\gamma^{1} * \gamma^{2}\right)$

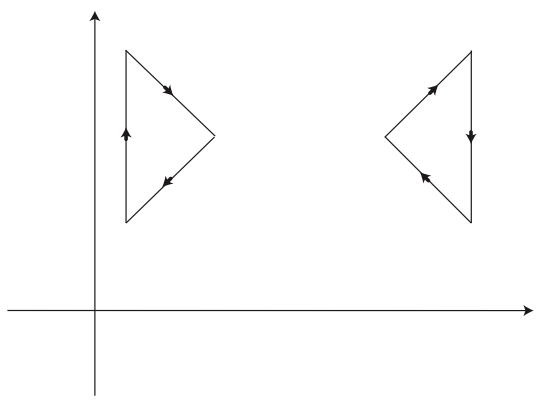

$S\left(\gamma^{\left.1 * \gamma^{2}\right)}\right.$

FiguRE 2. Here are pictured the image and the support of $\gamma^{1} * \gamma^{2}$.

Lemma 2.10. For $\delta>0$, if $p_{j}$ is a focal point, set:

$$
\Sigma_{\delta}:=\bigcup_{j=1}^{M} B_{\delta}\left(p_{j}\right)
$$

and

$$
S_{\delta}=\mathbb{R}^{2} \backslash \Sigma_{\delta}
$$

Let $P \in \mathcal{P}$ be a polygon such that, for every $\delta>0, V\left(P \cap S_{\delta}\right)$ is finite. Then there exists a polygon $P^{\delta} \in \mathcal{P}$ such that:

- $P^{\delta} \equiv P$ on $S_{\delta}$;

- $P^{\delta}$ has a finite number of vertices;

- the vertices of $P^{\delta}$ in $\Sigma_{\delta}$ have multiplicity 1 .

For the proof of this lemma see the Appendix.

Remark 2.11. If $P \in \mathcal{P}$ has a finite number of vertices, then there exists at least a $\Upsilon \in \tilde{\mathcal{C}}$ such that $S(\Upsilon)=\partial P$. Note that even if $P$ has only vertices with multiplicity 1 and is connected, there exist more than one $\Upsilon$ with this property.

Definition 2.12. For $P \in \mathcal{P}$, let $\Upsilon \in \tilde{\mathcal{C}}$ be such that $S(\Upsilon)=\partial P$. We say that $S(\Upsilon)$ is an ordered path of $\partial P$.

Remark 2.13. Let $P$ be an admissible polygon with a finite number of vertices such that there exists a sequence of sets $\left\{E_{n}\right\} \subset \mathcal{A}$ converging to $P$ with $\partial E_{n}$ converging to $\partial P$ uniformly.

Then there exists $\Upsilon=\left[\Upsilon^{1}, \ldots, \Upsilon^{m}\right] \in \tilde{\complement}$ (with $\Upsilon^{\alpha}=\left\{\gamma^{\alpha, 1}, \ldots, \gamma^{\alpha, k}\right\}$ for $\alpha \in\{1, \ldots, m\}$ ) such that:

- $S(\Upsilon)=\partial P$

- for each $\alpha \in\{1, \ldots, m\} \gamma^{\alpha}=\gamma^{\alpha, 1} * \ldots * \gamma^{\alpha, k}$ is closed (possibly with vertices of multiplicity greater than 1 , but without segments which transversally intersect);

- for all $\alpha \in\{1, \ldots, m\}$ and for all $i \in\{1, \ldots, n\} \gamma^{\alpha, i}$ has vertices only with multiplicity 1 .

Definition 2.14. Let $P$ be an admissible polygon and $Y$ an element of $\tilde{C}$ with the properties stated in Remark 2.13. We say that $S(\Upsilon)$ is an ordered path of $P$ induced by $\left\{E_{n}\right\}$.

Remark 2.15. Let $P$ be an admissible polygon with finite number of vertices. If $\Upsilon_{1}, \Upsilon_{2} \in \tilde{\mathcal{C}}$ are such that $S\left(\Upsilon_{1}\right)$ and $S\left(\Upsilon_{2}\right)$ are two ordered paths of $P$, then we do not necessarily have that $\operatorname{Im}\left(\Upsilon_{1}\right) \equiv \operatorname{Im}\left(\Upsilon_{2}\right)$. 


\section{The MAIN RESUlT}

Remark 3.1. In the sequel we identify $E \subset \mathbb{R}^{2}$ with its characteristic function $\chi_{E}$ and with a little abuse of notation we say that $\left\{E_{n}\right\}$ converges to $E$ (in $L^{1}\left(\mathbb{R}^{2}\right)$ ) if $\chi_{E_{n}} \rightarrow \chi_{E}$ in $L^{1}\left(\mathbb{R}^{2}\right)$.

Let $\varphi: \mathbb{R}^{2} \times S^{1} \rightarrow \mathbb{R}$ be the function defined as:

$$
\varphi(x, v)=\prod_{i=1}^{N}\left(v \cdot \tau_{i}^{\perp}\right)^{2} \prod_{j=1}^{M}\left(v \cdot\left(x-p_{j}\right)^{\perp}\right)^{2},
$$

where for $v_{1}, v_{2} \in \mathbb{R}^{2} v_{1} \cdot v_{2}$ denotes the usual scalar product in $\mathbb{R}^{2}$.

Remark 3.2. For each fixed $x, \varphi(x, \cdot)$ vanishes exactly on vectors parallel to an element of the set $D_{x}$ defined in (2.2).

Let $G_{\varepsilon}: \mathcal{A} \rightarrow[0,+\infty)$ be the functional defined as:

$$
G_{\varepsilon}(E)=\int_{\partial E}\left(\frac{\varphi(x, \tau)}{\varepsilon}+\varepsilon \kappa^{2}(x)\right) \mathrm{d} \mathcal{H}^{1}(x) .
$$

For $v_{1}, v_{2} \in S^{1}$ let $C\left(v_{1}, v_{2}\right)$ be the shortest of the two arcs in $S^{1}$ connecting $v_{1}$ and $v_{2}$. Let $g: \mathbb{R}^{2} \times S^{1} \times S^{1} \rightarrow$ $[0,+\infty)$ be defined as:

$$
g\left(x, v_{1}, v_{2}\right)=2 \int_{C\left(v_{1}, v_{2}\right)} \sqrt{\varphi(x, \xi)} \mathrm{d} \mathcal{H}^{1}(\xi) .
$$

For $P \in \mathcal{P}$ we define the following functional:

$$
G_{\mathcal{R}}(P)= \begin{cases}\sum_{p \in V(P) \backslash\left\{p_{1}, \ldots, p_{M}\right\}} g\left(p, v^{-}(p), v^{+}(p)\right) & \text { if } P \in \mathcal{R} \\ +\infty & \text { otherwise }\end{cases}
$$

where $v^{-}(p)$ and $v^{+}(p)$ denote the tangent vectors respectively on both sides of the point $p$ (considered in counterclockwise sense).

We denote by $G(P)$ the lower semicontinuous envelope of $G_{\mathcal{R}}$ with respect to the $L^{1}\left(\mathbb{R}^{2}\right)$-topology. We have the following formula for $G$ :

$$
G(P):=\inf \left\{\liminf _{n \rightarrow+\infty} G_{\mathcal{R}}\left(P_{n}\right): P_{n} \rightarrow P \text { in } L^{1}\left(\mathbb{R}^{2}\right)\right\} .
$$

Remark 3.3. The set on which the infimum in (3.5) is performed is not empty. In fact, given $P \in \mathcal{P}$, if for each $\delta>0$ we consider the polygon $P^{\delta}$ given by Lemma 2.10, to such a kind of polygon we can apply Remark 2.1 in [11], which ensures that there exists a sequence $\left\{R_{k}^{\delta}\right\} \subset \mathcal{R}$ converging in $L^{1}\left(\mathbb{R}^{2}\right)$ to $P^{\delta}$. Then the thesis follows by means of a diagonal argument.

For $\Upsilon=\left\{\gamma^{1}, \ldots, \gamma^{k}\right\} \in \mathcal{C}$ we define:

$$
F(\Upsilon)=\sum_{i=1}^{k} \sum_{l=1}^{n_{i}} g\left(q_{l}^{i}, v^{-}\left(q_{l}^{i}\right), v^{+}\left(q_{l}^{i}\right)\right)
$$

where we are assuming that each $\gamma^{i}$ has $n_{i}$ vertices, denoted by $q_{1}^{i}, \ldots, q_{n_{i}}^{i}$. 
For $\Upsilon=\left[\Upsilon^{1}, \ldots, \Upsilon^{m}\right] \in \tilde{\mathrm{e}}$, with a little abuse of notation we still denote by $F$ the functional defined as:

$$
F(\Upsilon):=\sum_{\alpha=1}^{m} F\left(\Upsilon^{\alpha}\right)
$$

In the sequel we shall need the following lemmata.

Lemma 3.4. Let $P \in \mathcal{P}$ be a polygon with $V(P)$ finite and let $\Upsilon \in \tilde{\mathcal{C}}$ be an ordered path of $P$ induced by a sequence $\left\{E_{n}\right\} \subset \mathcal{A}$. Then there exists a sequence of polygons $\left\{R_{h}\right\} \subset \mathcal{R}$ such that:

$$
R_{h} \rightarrow P \quad \text { and } \quad \lim _{h \rightarrow+\infty} G_{\mathcal{R}}\left(R_{h}\right) \leq F(\Upsilon)
$$

Proof. Let us denote by $\left\{q_{i}\right\}_{i=1, \ldots, k}$ the vertices of $\Upsilon$. We may use Lemma 3.4 in [11] (which gives the desired approximation for $P$, fixed the set of possible tangent direction of the approximating sets), applied by choosing such direction to be exactly the tangents to $P$. Hence we deduce that there exists an approximating sequence (of $P$ ) $\left\{R_{h}\right\} \subset \mathcal{R}$ such that, if we denote by $\left\{q_{i}^{h}\right\}_{i=1, \ldots, k_{h}}$ the vertices of $R_{h}$ (recall that $k_{h}$ can be grater or equal than $k$ ), for each $i \in\{1, \ldots, k\}$ there exists $l_{i} \in \mathbb{N}$ (with $\left.\sum_{i=1}^{k} l_{i}=k_{h}\right)$ and $\left\{q_{1}^{h}, \ldots, q_{l_{i}}^{h}\right\} \subset V\left(R_{h}\right)$ such that

$$
\lim _{h \rightarrow+\infty} q_{j}^{h}=q_{i} \quad \forall j \in\left\{1, \ldots, l_{i}\right\}
$$

and

$$
\sum_{i=1}^{k} \sum_{j=1}^{l_{i}} g\left(q_{i}, v_{j, h}^{+}, v_{j, h}^{-}\right) \leq F(\Upsilon)
$$

where $v_{j, h}^{+}$and $v_{j, h}^{-}$denote the directions of the two segments meeting in $q_{j}^{h}$. Using the continuity of $\varphi$ and recalling that $G_{\mathcal{R}}\left(R_{h}\right)=\sum_{i=1}^{k} \sum_{j=1}^{l_{i}} g\left(q_{j}^{h}, v_{j, h}^{+}, v_{j, h}^{-}\right)$, by (3.8) we argue:

$$
\lim _{h \rightarrow+\infty} G_{\mathcal{R}}\left(R_{h}\right)=\lim _{h \rightarrow+\infty} \sum_{i=1}^{k} \sum_{j=1}^{l_{i}} \varphi\left(q_{j}^{h}, v_{j, h}^{+}, v_{j, h}^{-}\right)=\sum_{i=1}^{k} g\left(q_{i}, v_{i}^{+}, v_{i}^{-}\right) \leq F(\Upsilon),
$$

which is the thesis.

Lemma 3.5. Let $\left\{\rho_{\varepsilon}\right\} \subset \mathbb{R}$ be a sequence such that $\rho_{\varepsilon}>0$ and $\rho_{\varepsilon}=o(\sqrt{\varepsilon})$. For $q \in \mathbb{R}^{2} \backslash\left\{p_{1}, \ldots, p_{M}\right\}$ consider $B_{\rho_{\varepsilon}}(q)$. For $\nu_{1}, \nu_{2} \in S^{1}$ and $\eta \in\left(0, \frac{L\left(\nu_{1}, \nu_{2}\right)}{2}\right)$, let $\alpha:[a, b] \rightarrow B_{\rho_{\varepsilon}}(q)$ be a curve of class $C^{2}$ parameterized by the arc length and such that $\alpha(a), \alpha(b) \in \partial B_{\rho_{\varepsilon}}(q)$ and $\dot{\alpha}:[a, b] \rightarrow C\left(\nu_{1}, \nu_{2}\right)$, with $\dot{\alpha}(a)=\nu_{1}+\eta, \dot{\alpha}(b)=\nu_{2}-\eta$. (The value $\dot{\alpha}(a)$ has to be thought as $\lim _{t \rightarrow a^{+}} \dot{\alpha}(t)$, and the same applies for $\dot{\alpha}(b)$.) Then the following inequality holds:

$$
\int_{a}^{b}\left(\frac{1}{\varepsilon} \varphi(\alpha(s), \dot{\alpha}(s))+\varepsilon|\ddot{\alpha}(s)|^{2}\right) \mathrm{d} s \geq g\left(q, \nu_{1}, \nu_{2}\right)+o_{\varepsilon}(1)+o_{\eta}(1) .
$$

For the proof of this lemma see the Appendix.

The first theorem we prove is the following $\Gamma$-convergence result. 
Theorem 3.6 (Г-convergence). Let $\widetilde{G}_{\varepsilon}, \widetilde{G}: L^{1}\left(\mathbb{R}^{2}\right) \rightarrow[0,+\infty]$ be the functionals given by:

$$
\begin{gathered}
\widetilde{G}_{\varepsilon}(u)= \begin{cases}G_{\varepsilon}(E) & \text { if } u=\chi_{E} \text { with } \partial E \text { of class } C^{2} \\
+\infty & \text { otherwise, }\end{cases} \\
\widetilde{G}(u)= \begin{cases}G(P) & \text { if } u=\chi_{P} \text { with } P \in \mathcal{P} \\
+\infty & \text { otherwise. }\end{cases}
\end{gathered}
$$

Then $\left\{\widetilde{G}_{\varepsilon}\right\} \Gamma$-converges to $\widetilde{G}$ with respect to the $L^{1}\left(\mathbb{R}^{2}\right)$-topology.

Proof. As already focused in Remark 3.1, we recall that for the sake of simplicity we identify functionals defined on sets and functionals defined on characteristic functions of sets. We perform this proof (as well as that of Thm. 3.14) directly taking in account the functionals $G_{\varepsilon}$ and $G$.

We first prove the $\Gamma$-liminf inequality. Let $P$ be an admissible polygon and let $\left\{E_{n}\right\} \subset A$ be a family of sets converging to $P$ in $L^{1}\left(\mathbb{R}^{n}\right)$ and such that $\liminf _{n \rightarrow+\infty} G_{\varepsilon_{n}}\left(E_{n}\right)<+\infty$. We want to prove that

$$
G(P) \leq \liminf _{n \rightarrow+\infty} G_{\varepsilon_{n}}\left(E_{n}\right) .
$$

Let $P_{\delta}$ be the polygon given by the construction performed in Lemma 2.10 and denote by $\left\{E_{n}^{\delta}\right\}$ a sequence of subsets of $\mathcal{A}$ such that $E_{n}^{\delta}$ coincides with $E_{n}$ in $S_{\delta}, E_{n}^{\delta}$ converges to $P^{\delta}$ in $L^{1}\left(\mathbb{R}^{2}\right)$ (the construction of such a kind of sets can be performed as follows: for each pair $q_{i}, q_{i+1}$ of points belonging to the set $\mathcal{U}$ defined in the proof of Lemma 2.10, consider for instance an extension of $P^{\delta}$ of the type $\left[q_{i}, h_{i}\right] \cup\left[h_{i}, q_{i+1}\right]$, where $h_{i}$ is the suitable point given by the same lemma; then we can consider a $C^{1}$-curve converging uniformly to $\left[q_{i}, h_{i}\right] \cup\left[h_{i}, q_{i+1}\right]$ and smoothly joining the curve $E_{n} \cap S_{\delta}$ in $q_{i}$ and $q_{i+1}$; finally we take as extension of $E_{n}^{\delta}$ in $\Sigma_{\delta}$ the union of all these $C^{1}$-curves). Let $\Upsilon^{\delta}$ be an ordered path of $P^{\delta}$ induced by $\left\{E_{n}^{\delta}\right\}$. By Lemma 3.5 we have:

$$
\begin{gathered}
F\left(\Upsilon^{\delta} \cap S_{\delta}\right) \leq G_{\varepsilon_{n}}\left(E_{n}\right), \\
F\left(\Upsilon^{\delta}\right)=F\left(\Upsilon^{\delta} \cap S_{\delta}\right)+\xi_{\delta}, \quad \text { with } \quad \xi_{\delta}=o_{\delta}(1)
\end{gathered}
$$

and therefore

$$
F\left(\Upsilon^{\delta}\right) \leq G_{\varepsilon_{n}}\left(E_{n}\right)+\xi_{\delta} .
$$

Let $\left\{R_{h}^{\delta}\right\} \subset \mathcal{R}$ be the sequence given by Lemma 3.4 for $\Upsilon^{\delta}$. Recalling the definition of $G$ in (3.5), we immediately get:

$$
G\left(P^{\delta}\right) \leq G_{\varepsilon_{n}}\left(E_{n}\right)+\xi_{\delta} .
$$

If we choose a sequence $\left\{\delta_{n}\right\}$ converging to 0 as $n$ goes to infinity, by construction of $P^{\delta}$ we know that $P^{\delta_{n}}$ converges to $P$ in $L^{1}\left(\mathbb{R}^{2}\right)$ and, since by definition $G$ is lower semicontinuous, we argue that $G(P) \leq \liminf _{n \rightarrow+\infty} G\left(P^{\delta}\right)$. Hence, using a diagonal argument and passing to the limit for $n$ going to infinity in (3.16), we get the desired inequality (3.12).

We now prove the $\Gamma$-limsup inequality, that means: for each admissible polygon $P$ we want to find a sequence $\left\{E_{n}\right\} \subset \mathcal{A}$ converging to $P$ in $L^{1}\left(\mathbb{R}^{2}\right)$ and such that

$$
\limsup _{n \rightarrow+\infty} G_{\varepsilon_{n}}\left(E_{n}\right) \leq G(P) .
$$

We proceed in two steps: we first prove inequality (3.17) for regular polygons and then we extend the result to a general admissible polygon by means of an approximation lemma.

Let $R$ be a regular polygon. Without loss of generality we can assume that $\partial R$ is connected. Let $\left\{q_{1}, \ldots, q_{k}\right\}$ be the elements of $V(R)$ and let us assume that they are ordered running through $\partial R$ in counterclockwise sense. 
Recall that by definition of regular polygon, $k$ is finite. For $q_{i} \in V(R)$ define the segment $s_{i}=\left[q_{i}, q_{i+1}\right]$ and let $L_{i}$ be the length of $s_{i}$. If

$$
v_{1}^{i}:=\frac{q_{i}-q_{i-1}}{\left|q_{i}-q_{i-1}\right|} \quad \text { and } \quad v_{2}^{i}:=\frac{q_{i+1}-q_{i}}{\left|q_{i+1}-q_{i}\right|}
$$

are the two unit vectors of the directions which meet at $q_{i}$, define the curve $\gamma^{i}:\left[-\frac{L_{i-1}}{2}, \frac{L_{i}}{2}\right] \rightarrow \mathbb{R}^{2}$ in the following way:

$$
\gamma^{i}(t)= \begin{cases}q_{i}-t v_{1}^{i} & t \in\left[-\frac{L_{i-1}}{2}, 0\right] \\ q_{i}+t v_{2}^{i} & t \in\left[0, \frac{L_{i}}{2}\right] .\end{cases}
$$

Remark 3.7. It can be immediately checked that $\gamma:=\gamma^{1} * \ldots * \gamma^{k}$ is a parameterization of $\partial P$.

Remark 3.8. By construction we have that $v_{1}^{i}$ and $v_{2}^{i}$ belong to $D_{q_{i}}$, that means: $\varphi\left(q_{i}, v_{1}^{i}\right)=\varphi\left(q_{i}, v_{2}^{i}\right)=0$.

We construct an approximating sequence $\left\{\Gamma_{n}\right\}$ of $\partial P$ in such a way that $\gamma_{n}$ (parameterization of $\Gamma_{n}$ ) is $C^{2}$ and in the form:

$$
\gamma_{n}=\gamma_{n}^{1} * \ldots * \gamma_{n}^{k}
$$

with $\gamma_{n}^{i}$ converging to $\gamma^{i}$ uniformly. Let $E_{n}$ be the domain included by $\Gamma_{n}$.

Definition 3.9. Let $C$ be a curve parameterized by $\beta:[a, b] \rightarrow \mathbb{R}^{2}$. For $x_{1}, x_{2} \in C$, we say that " $x_{1}$ precedes $x_{2}$ " (and we write " $x_{1} \prec x_{2}$ ") if $x_{1}=\beta\left(t_{1}\right)$ and $x_{2}=\beta\left(t_{2}\right)$ with $t_{1}<t_{2}$.

Definition 3.10. Let $\lambda: \mathbb{R} \rightarrow S^{1}$ be the usual parameterization:

$$
\lambda(\vartheta)=(\cos (\vartheta), \sin (\vartheta)),
$$

useful to identify $S^{1}$ with $\mathbb{R}$ modulo $2 \pi$.

Let $C$ be an $S^{1}$-valued function. We say that $C$ is $S^{1}$-increasing if, for every $x_{1}, x_{2} \in C$ with $x_{1} \prec x_{2}$, it holds: $\lambda^{-1}\left(x_{1}\right)<\lambda^{-1}\left(x_{2}\right)$.

Definition 3.11. For $\nu_{1}, \nu_{2} \in S^{1}$ let $\theta_{1}, \theta_{2} \in \mathbb{R}$ modulo $2 \pi$ be the respective angle. We define $m\left(\nu_{1} \nu_{2}\right):=$ $\left(\cos \left(\frac{\theta_{1}+\theta_{2}}{2}\right), \sin \left(\frac{\theta_{1}+\theta_{2}}{2}\right)\right)$.

In the next proposition we construct the optimal profile $\gamma_{n}^{i}$.

Proposition 3.12. Let $\Gamma^{i}$ be the curve parameterized by $\gamma^{i}:\left[-\frac{L_{i}}{2}, \frac{L_{i+1}}{2}\right] \rightarrow \mathbb{R}^{2}$ defined as in (3.18).

Then, for every positive sequence $\left\{\varepsilon_{n}\right\}$ converging to 0 , there exists a sequence $\left\{\Gamma_{n}^{i}\right\}$ of curves parameterized by $\gamma_{n}^{i}:\left[-\frac{L_{i}}{2}, \frac{L_{i+1}}{2}\right] \rightarrow \mathbb{R}^{2}$ such that:

(1) $\gamma_{n}^{i} \in C^{2}$;

(2) $\gamma_{n}^{i}\left(-\frac{L_{i}}{2}\right)=\gamma^{i}\left(-\frac{L_{i}}{2}\right)$ and $\gamma_{n}^{i}\left(\frac{L_{i+1}}{2}\right)=\gamma^{i}\left(\frac{L_{i+1}}{2}\right)$;

(3) $\gamma_{n}^{i} \rightarrow \gamma^{i}$ uniformly;

(4) $\int_{\Gamma_{n}^{i}}\left[\frac{1}{\varepsilon_{n}} \varphi(x, \tau(x))+\varepsilon_{n} \kappa^{2}(x)\right] \mathrm{d} \mathcal{H}^{1}(x) \leq g\left(q^{i}, v_{1}^{i}, v_{2}^{i}\right)+o_{\varepsilon_{n}}(1)$.

Proof. Let $\left\{\varepsilon_{n}\right\} \subset \mathbb{R}$ be a positive sequence converging to 0 . For $n$ large enough we can suppose that $2 \varepsilon_{n}$ belongs to the interval $\left(0, \min \left\{\frac{L_{i}}{2}, \frac{L_{i+1}}{2}\right\}\right)$. We divide the interval $\left[-\frac{L_{i}}{2}, \frac{L_{i+1}}{2}\right]$ in the following five intervals:

$$
\begin{gathered}
I_{1}^{i, n}=\left[-\frac{L_{i}}{2},-\varepsilon_{n}-\varepsilon_{n}^{2}\right], \quad I_{2}^{i, n}=\left[-\varepsilon_{n}-\varepsilon_{n}^{2},-\varepsilon_{n}\right], \quad I_{3}^{i, n}=\left[-\varepsilon_{n}, \varepsilon_{n}\right], \\
I_{4}^{i, n}=\left[\varepsilon_{n}, \varepsilon_{n}+\varepsilon_{n}^{2}\right], \quad I_{5}^{i, n}=\left[\varepsilon_{n}+\varepsilon_{n}^{2}, \frac{L_{i+1}}{2}\right]
\end{gathered}
$$

and we choose a suitable definition of $\gamma_{n}^{i}$ in each $I_{j}^{i, n}$. 
To do this, we consider the problem of finding a solution $u: \mathbb{R} \rightarrow S^{1}$ of the following Cauchy problem:

$$
\left\{\begin{array}{l}
\dot{u}(t)=\sqrt{\varphi\left(q_{i}, u(t)\right)} J u(t), \\
u(0)=m\left(v_{1}^{i}, v_{2}^{i}\right),
\end{array}\right.
$$

where $J$ is the $\pi / 2$-rotation matrix defined in Section 2. By the regularity properties of $\varphi$, classical results (on ordinary differential equations) ensure that there exists a $C^{1}$ and unique maximal solution of (3.19). Moreover $|u(t)|$ has to be constant, because $\frac{\mathrm{d}}{\mathrm{d} t}|u(t)|^{2}=2 u(t) \cdot \dot{u}(t)=2 \sqrt{\varphi\left(q_{i}, u(t)\right)} u(t) \cdot u(t)^{\perp}=0$ and, since $|u(0)|=1$, we immediately get:

$$
u(t) \in S^{1} \quad \forall t \in \mathbb{R} .
$$

If we rewrite equation (3.19) in the integral form:

$$
u(t)=m\left(v_{1}^{i}, v_{2}^{i}\right)+\int_{0}^{t} \sqrt{\varphi\left(q_{i}, u(\sigma)\right)} u(\sigma)^{\perp} d \sigma
$$

recalling that we denote by $u(\sigma)^{\perp}$ a rotation of $\frac{\pi}{2}$ in counterclockwise sense, we get that $u$ is $S^{1}$-increasing. We define

$$
\beta_{n}(t):=q_{i}+\int_{0}^{t} u\left(\frac{\sigma}{\varepsilon}\right) \mathrm{d} \sigma
$$

which by construction is $C^{2}$ and parameterized by the arc length.

We define $\gamma_{n}^{i}:\left[-\frac{L_{i}}{2}, \frac{L_{i+1}}{2}\right] \rightarrow \mathbb{R}^{2}$ in the following form:

$$
\gamma_{n}^{i}(t)= \begin{cases}v_{1}^{i} & \text { if } t \in I_{1}^{i, n} \\ \psi_{n}^{1}(t) & \text { if } t \in I_{2}^{i, n} \\ \beta_{n}(t) & \text { if } t \in I_{3}^{i, n} \\ \psi_{n}^{2} & \text { if } t \in I_{4}^{i, n} \\ v_{2}^{i} & \text { if } t \in I_{5}^{i, n}\end{cases}
$$

where $\psi_{n}^{1}(t): I_{2}^{i, n} \rightarrow \mathbb{R}^{2}$ and $\psi_{n}^{2}(t): I_{4}^{i, n} \rightarrow \mathbb{R}^{2}$ are $C^{2}$ and such that:

$$
\left\{\begin{array} { l } 
{ \ddot { \psi } _ { n } ^ { 1 } ( - \varepsilon _ { n } - \varepsilon _ { n } ^ { 2 } ) = 0 } \\
{ \dot { \psi } _ { n } ^ { 1 } ( - \varepsilon _ { n } - \varepsilon _ { n } ^ { 2 } ) = \nu _ { 1 } ^ { i } } \\
{ \dot { \psi } _ { n } ^ { 1 } ( - \varepsilon _ { n } ) = \beta _ { n } ( - \varepsilon _ { n } ) , }
\end{array} \quad \left\{\begin{array}{l}
\ddot{\psi}_{n}^{2}\left(\varepsilon_{n}+\varepsilon_{n}^{2}\right)=0 \\
\dot{\psi}_{n}^{2}\left(\varepsilon_{n}+\varepsilon_{n}^{2}\right)=\nu_{1}^{i} \\
\dot{\psi}_{n}^{2}\left(\varepsilon_{n}\right)=\beta_{n}\left(\varepsilon_{n}\right)
\end{array}\right.\right.
$$

and

$$
\left\{\begin{array}{l}
\left|\dot{\psi}_{n}^{2}(t)\right|<2\left|\dot{\beta}_{n}\left(-\varepsilon_{n}\right)-v_{1}^{i}\right| \quad \forall t \in I_{2}^{i, n} \\
\left|\dot{\psi}_{n}^{2}(t)\right|<2\left|\dot{\beta}_{n}\left(-\varepsilon_{n}\right)-v_{1}^{i}\right| \quad \forall t \in I_{2}^{i, n}
\end{array}\right.
$$


By construction $\gamma_{n}^{i}$ satisfies properties (1)-(3). Let us now check property (4). We have:

$$
\begin{aligned}
& \int_{\Gamma_{n}^{i}}\left[\frac{1}{\varepsilon_{n}} \varphi(x, \tau(x))+\varepsilon_{n} \kappa^{2}(x)\right] \mathrm{d} \mathcal{H}^{1}(x)= \\
& \int_{\Gamma_{n}^{i}}\left[\frac{1}{\varepsilon_{n}} \varphi\left(q_{i}, \tau(x)\right)+\varepsilon_{n} \kappa^{2}(x)\right] \mathrm{d} \mathcal{H}^{1}(x)+\int_{\Gamma_{n}^{i}} \frac{1}{\varepsilon_{n}} \nabla_{x} \varphi\left(q_{i}, \tau(x)\right) \cdot\left(\zeta_{x}-q_{i}\right) \mathrm{d} \mathcal{H}^{1}(x),
\end{aligned}
$$

where $\zeta_{x} \in\left(\gamma_{n}^{i}(x), q_{i}\right)$. Let us denote by $\mathcal{G}_{n}^{1}$ and $\mathcal{G}_{n}^{2}$ respectively the first and the second term in (3.26). We recall that by definition of $\varphi$ we know that there exists $C_{1}>0$ such that $\left|\nabla_{x} \varphi\left(q_{i}, \tau(x)\right)\right| \leq C_{1}$ for every $x \in \Gamma_{n}^{i}$. Moreover by the properties of $\beta_{n}, \psi_{n}^{1}$ and $\psi_{n}^{2}$ we have: $\left|\zeta_{x}-q_{i}\right| \leq C_{2} \varepsilon_{n}$, for suitable $C_{2}>0$. Finally, denoted by $\tilde{\Gamma}_{n}^{i}$ the support of $\psi_{n}^{1} * \beta_{n} * \psi_{n}^{2}$, then we get:

$$
\begin{aligned}
\left|\mathcal{G}_{n}^{2}\right| & =\left|\int_{\Gamma_{n}^{i}} \frac{1}{\varepsilon_{n}} \nabla_{x} \varphi\left(q_{i}, \tau(x)\right) \cdot\left(\zeta_{x}-q_{i}\right) \mathrm{d} \mathcal{H}^{1}(x)\right| \\
& \leq \int_{\tilde{\Gamma}_{n}^{i}} \frac{1}{\varepsilon_{n}}\left|\nabla_{x} \varphi\left(q_{i}, \tau(x)\right)\right|\left|\zeta_{x}-q_{i}\right| \mathrm{d} \mathcal{H}^{1}(x) \leq C_{1} C_{2} \varepsilon_{n} .
\end{aligned}
$$

Let us estimate the quantity $\mathcal{G}_{n}^{1}$ :

$$
\begin{aligned}
\mathcal{G}_{n}^{1}= & \int_{-\frac{L_{i}}{2}}^{\frac{L_{i+1}}{2}}\left[\frac{1}{\varepsilon_{n}} \varphi\left(q_{i}, \dot{\gamma}_{n}^{i}(t)\right)+\varepsilon_{n}\left|\ddot{\gamma}_{n}^{i}(t)\right|\right] \mathrm{d} t \\
= & \int_{I_{1}^{i, n} \cup I_{5}^{i, n}}\left[\frac{1}{\varepsilon_{n}} \varphi\left(q_{i}, \dot{\gamma}_{n}^{i}(t)\right)+\varepsilon_{n}\left|\ddot{\gamma}_{n}^{i}(t)\right|\right] \mathrm{d} t+\int_{I_{2}^{i, n} \cup I_{4}^{i, n}}\left[\frac{1}{\varepsilon_{n}} \varphi\left(q_{i}, \dot{\gamma}_{n}^{i}(t)\right)+\varepsilon_{n}\left|\ddot{\gamma}_{n}^{i}(t)\right|\right] \mathrm{d} t \\
& \quad+\int_{I_{3}^{i, n}}\left[\frac{1}{\varepsilon_{n}} \varphi\left(q_{i}, \dot{\gamma}_{n}^{i}(t)\right)+\varepsilon_{n}\left|\ddot{\gamma}_{n}^{i}(t)\right|\right] \mathrm{d} t .
\end{aligned}
$$

Recalling Remark 3.8 and the definition of $\gamma_{n}^{i}$, we have that the first term in the latter sum of equation (3.28) is trivially equal to 0 and we can rewrite:

$$
\begin{aligned}
\mathcal{G}_{n}^{1}= & \int_{-\varepsilon_{n}-\varepsilon_{n}^{2}}^{-\varepsilon_{n}}\left[\frac{1}{\varepsilon_{n}} \varphi\left(q_{i}, \dot{\psi}_{n}^{1}(t)\right)+\varepsilon_{n}\left|\ddot{\psi}_{n}^{1}(t)\right|\right] \mathrm{d} t+\int_{\varepsilon_{n}}^{\varepsilon_{n}+\varepsilon_{n}^{2}}\left[\frac{1}{\varepsilon_{n}} \varphi\left(q_{i}, \dot{\psi}_{n}^{2}(t)\right)+\varepsilon_{n}\left|\ddot{\psi}_{n}^{2}(t)\right|\right] \mathrm{d} t \\
& +\int_{-\varepsilon_{n}}^{\varepsilon_{n}}\left[\frac{1}{\varepsilon_{n}} \varphi\left(q_{i}, \dot{\beta}_{n}(t)\right)+\varepsilon_{n}\left|\ddot{\beta}_{n}(t)\right|\right] \mathrm{d} t .
\end{aligned}
$$

Moreover, by property (3.25) of $\psi_{n}^{1}$ and $\psi_{n}^{2}$ and recalling the differential property satisfied by the derivative of $\beta_{n}$, for suitable $M>0$ we get:

$$
\left|\mathcal{G}_{n}^{1}\right| \leq M \varepsilon_{n}+\mu \varepsilon_{n}^{3}+g\left(q_{i}, u(-1), u(1)\right) \leq o\left(\varepsilon_{n}\right)+g\left(q_{i}, v_{1}^{i}, v_{2}^{i}\right),
$$

where we have set $\mu:=\left|u(1)-v_{1}^{i}\right|^{2}+\left|u(-1)-v_{2}^{i}\right|^{2}$. By (3.26), (3.27) and (3.30) we finally get the thesis.

Now we are in position to prove inequality (3.17) for a regular polygon. In fact, as already said above, we take $E_{n}$ as the interior of the curve $\Gamma_{n}$ parameterized by $\gamma_{n}:=\gamma_{n}^{1} * \ldots * \gamma_{n}^{k}$ and we get:

$$
\begin{aligned}
G_{\varepsilon_{n}}\left(E_{n}\right) & =\sum_{i=1}^{k} \int_{\Gamma_{n}^{i}}\left[\frac{1}{\varepsilon_{n}} \varphi(x, \tau(x))+\varepsilon_{n} \kappa^{2}(x)\right] \mathrm{d} \mathcal{H}^{1}(x) \leq \sum_{i=1}^{k} g\left(q^{i}, v_{1}^{i}, v_{2}^{i}\right)+o\left(\varepsilon_{n}\right) \\
& =G_{\mathcal{R}}(P)+o\left(\varepsilon_{n}\right) \equiv G(P)+o\left(\varepsilon_{n}\right)
\end{aligned}
$$

and, passing to the limsup for $n$ tending to $+\infty$, we get the thesis. 
Now we consider an admissible polygon $P$ such that $G(P)<+\infty$. To prove inequality (3.17) for this kind of polygon we need the following lemma.

Lemma 3.13. For each $P \in \mathcal{P}$ such that $G(P)<+\infty$ there exists a sequence $\left\{R_{k}\right\} \subset \mathcal{R}$ converging to $P$ in $L^{1}\left(\mathbb{R}^{2}\right)$ and such that

$$
\lim _{k \rightarrow+\infty} G_{\mathcal{R}}\left(R_{k}\right)=G(P) .
$$

To prove this lemma we can take, for each $\delta>0$, the polygon $P^{\delta}$ given by Lemma 2.10. To this kind of polygon we can apply Remark 2.2 in [11], which ensures (using the continuity of $\varphi$ ) that there exists a sequence $\left\{R_{k}^{\delta}\right\} \subset \mathcal{R}$ converging to $P^{\delta}$ in $L^{1}\left(\mathbb{R}^{2}\right)$ and such that (3.32) holds. Then by means of a diagonal argument we recover an approximating sequence for $P$. Moreover, since $P^{\delta}$ has no vertices in $\left\{p_{1}, \ldots, p_{M}\right\}$, we can assume the same property for the approximating sequence $R_{k}$, even thought this is not strictly necessary, and the proof could work the same (with some minor modification) also without this requirement.

We can now conclude the proof of Theorem 3.6. Let $\left\{R_{k}\right\}$ be the sequence given by Lemma 3.13. Since $R_{k}$ is regular, we have already proved that there exists a sequence $\left\{E_{n}^{k}\right\} \subset \mathcal{A}$ such that:

$$
\limsup _{n \rightarrow+\infty} G_{\varepsilon_{n}}\left(E_{n}^{k}\right) \leq G_{\mathcal{R}}\left(R_{k}\right) .
$$

Hence by (3.32) and (3.33), we can choose a suitable subsequence of $\left\{E_{n}^{k}\right\}$ converging to $P$ in $L^{1}\left(\mathbb{R}^{2}\right)$ and with the desired property to fulfill (3.17).

In the following theorem we give a compactness result under the additional assumption of equi-boundedness of perimeters.

Theorem 3.14 (compactness). Let $\left\{E_{n}\right\} \subset \mathcal{A}$ be such that

$$
\begin{aligned}
& \sup _{n} \mathcal{H}^{1}\left(\partial E_{n}\right)=M_{1}<+\infty \\
& \sup _{n} G_{\varepsilon_{n}}\left(E_{n}\right)=M_{2}<+\infty
\end{aligned}
$$

and

$$
\text { there exists a bounded } K \subset \mathbb{R}^{2} \text { such that } E_{n} \subset K \text { for all } n \text {. }
$$

Then there exist a subsequence $\left\{E_{n_{k}}\right\}$ and $P \in \mathcal{P}$ such that: $E_{n_{k}} \rightarrow P$ in $L^{1}\left(\mathbb{R}^{2}\right)$ and $G(P)<+\infty$.

Proof. Let $K$ be the number (possibly equal to $+\infty$ ) of connected components of $\partial E_{n}$ (passing to a subsequence we can suppose that $K$ is independent of $n$ ). We denote by $\Gamma_{n}^{i}$ each connected component of $\partial E_{n}$.

Remark 3.15. We assume that the boundary of $\partial E_{n}$ is oriented in such a way that the outer connected component is oriented in counterclockwise sense.

Let $\left\{\gamma_{n}^{i}\right\} \subset C^{2}\left([0,1] ; \mathbb{R}^{2}\right)$ be a sequence of parameterizations of $\Gamma_{n}^{i}$. We suppose that these curves are parameterized proportionally to their arc length, which means there exists $\left\{c_{n}^{i}\right\} \subset \mathbb{R}$ such that $\left|\dot{\gamma}_{n}(t)\right|=c_{n}^{i}$ $\forall t \in[0,1]$. By assumption (3.34) we immediately get that $\left\{c_{n}^{i}\right\}$ is bounded and hence for each $i$ we have:

$$
\sup _{n} \int_{0}^{1}\left|\dot{\gamma}_{n}^{i}(t)\right|^{2} \mathrm{~d} t<+\infty .
$$

On the other hand we also have:

$$
\sup _{n} \int_{0}^{1}\left|\gamma_{n}^{i}(t)\right|^{2} \mathrm{~d} t<+\infty
$$


This follows by the following inequality:

$$
\left|\gamma_{n}^{i}(t)\right| \leq\left|\gamma_{n}^{i}(0)\right|+\int_{0}^{t}\left|\dot{\gamma}_{n}^{i}(\sigma)\right| \mathrm{d} \sigma \leq\left|\gamma_{n}^{i}(0)\right|+M_{1}
$$

and by hypotheses (3.36) we may assume: $\sup _{n}\left|\gamma_{n}^{i}(0)\right|<+\infty$. By (3.37) and (3.38) we conclude that $\left\{\gamma_{n}^{i}\right\}$ is bounded in $W^{1,2}\left([0,1] ; \mathbb{R}^{2}\right)$. Since the immersion of $W^{1,2}\left([0,1] ; \mathbb{R}^{2}\right)$ in $C^{0}\left([0,1] ; \mathbb{R}^{2}\right)$ is compact, we immediately deduce that there exists a subsequence $\left\{\gamma_{n_{k}}^{i}\right\}$ uniformly converging to a $\gamma^{i} \in C^{0}\left([0,1] ; \mathbb{R}^{2}\right)$. We denote by $\Gamma^{i}$ the curve parameterized by $\gamma^{i}$.

If $\Gamma$ is a curve parameterized by $\gamma \in C^{2}\left([0,1] ; \mathbb{R}^{2}\right)$, for $\xi=\left(\xi_{1}, \xi_{2}\right) \in \mathbb{R}^{2} \backslash \Gamma$ we denote by $\sigma(\gamma, \xi)$ the winding number of $\gamma$ around $\xi$ defined as:

$$
\sigma(\gamma, \xi):=\int_{0}^{1}\left(-\frac{\gamma_{2}(t)-\xi_{2}}{\left(\gamma_{1}(t)-\xi_{1}\right)^{2}+\left(\gamma_{2}(t)-\xi_{2}\right)^{2}} \dot{\gamma}_{1}(t)+\frac{\gamma_{1}(t)-\xi_{1}}{\left(\gamma_{1}(t)-\xi_{1}\right)^{2}+\left(\gamma_{2}(t)-\xi_{2}\right)^{2}} \dot{\gamma}_{2}(t)\right) \mathrm{d} t
$$

Without loss of generality we may assume that $\sigma\left(\gamma_{n}^{i}, \xi\right) \in\{-1,0,1\}$ for all $\xi \in \mathbb{R}^{2} \backslash \Gamma_{n}^{i}, n \in \mathbb{N}, i \in\{1, \ldots, K\}$. Recalling that $\sigma(\cdot, \xi)$ is continuous with respect to the uniform convergence, we can extend the definition of $\sigma$ to $\gamma^{i}$, in such a way that: $\sigma\left(\gamma^{i}, \xi\right)=\lim _{n \rightarrow+\infty} \sigma\left(\gamma_{n}^{i}, \xi\right)$. In particular, since the winding number is integer-valued, there exists $\bar{n} \in \mathbb{N}$ such that $\sigma\left(\gamma^{i}, \xi\right)=\sigma\left(\gamma_{n}^{i}, \xi\right)$ for every $n>\bar{n}$ and for every $\xi \in \mathbb{R}^{2} \backslash \cup_{i=1}^{K} \Gamma^{i}$. Now we define:

$$
P:=\left\{\xi \in \mathbb{R}^{2} \backslash \bigcup_{i=1}^{K} \Gamma^{i}: \sum_{i=1}^{K} \sigma\left(\gamma^{i}, \xi\right)=1\right\}
$$

Remark 3.16. The function $\chi(\xi):=\sum_{i=1}^{K} \sigma\left(\gamma^{i}, \xi\right)$ takes only the values 0 or 1 . If $K$ is finite, this immediately follows by definition of $\sigma$ and Remark 3.15. If instead $K$ is equal to $+\infty$, then there exists a focal point $p$ such that $d^{i}:=\sup _{t \in[0,1]}\left|\gamma^{i}(t)-p\right|$ goes to 0 as $i$ tends to $+\infty$. Therefore, if we set $l:=|\xi-p|$, we have that there exists $\bar{i} \in \mathbb{N}$ such that $d^{i}<l$ for every $i>\bar{i}$ and hence $\sigma\left(\gamma^{i}, \xi\right)$ is equal to 0 for every $i>\bar{i}$.

Remark 3.17. By construction we have that: $\partial P=\bigcup_{i=1}^{K} \Gamma^{i}$.

To conclude the proof, we need to show that $P$ is an admissible polygon. Let $\Gamma_{n_{k}}^{i}$ be the curve parameterized by $\gamma_{n_{k}}^{i}$. Using (3.35), we get:

$$
\int_{\Gamma_{n_{k}}^{i}} \varphi(x, \tau(x)) \mathrm{d} \mathcal{H}^{1}(x) \leq \varepsilon_{n} M_{2} \quad \forall \varepsilon_{n}>0
$$

and hence, for $\varepsilon_{n_{k}}$ going to $0, \Gamma_{n_{k}}^{i}$ converges pointwise to a curve such that its tangent vector $\tau(x)$ belongs to $D_{x}$ for a.e. $x \in \Gamma^{i}$.

We now show that the cardinality of $V(P)$ is at most countable and $G$ is finite on $P$. For $\delta>0$, consider the set $S_{\delta}$ defined in (2.4). Recalling the definition of $\varphi$ given above, for every $\delta>0$ there exists $c_{\delta}>0$ (with $\left.c_{\delta}=o_{\delta}(1)\right)$ such that $g\left(p, v^{-}(p), v^{+}(p)\right) \geq c_{\delta}$ for all $p \in S_{\delta}$. Therefore we argue that $\bigcup_{i=1}^{K} \Gamma^{i}$ must have a finite number of vertices in $S_{\delta}$. For $\delta$ going to 0 , by a diagonal argument we get that $E_{n_{k}}$ tends to an admissible polygon $P$ with (possibly) countable vertices near the focal points.

Note that by (3.35) and (3.9) and the definition of $G$, it immediately follows that $G$ is finite on $P$. 

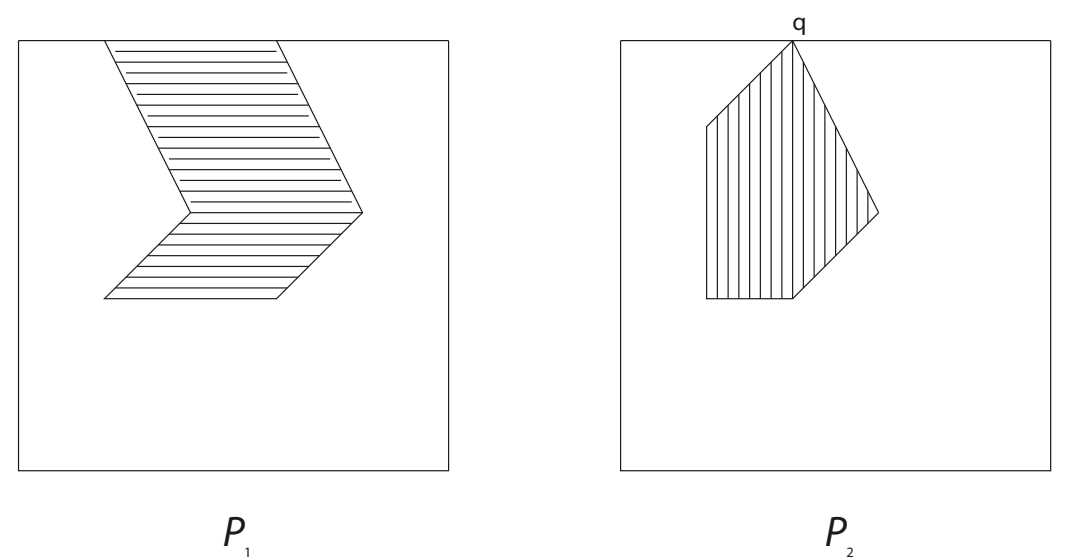

Figure 3. $P_{1}$ is such that $V\left(P_{1}\right)=\tilde{V}\left(P_{1}\right)$, while $V\left(P_{2}\right)$ is strictly contained in $\tilde{V}\left(P_{2}\right)$; in fact the point $q$ belongs to $\tilde{V}(P) \backslash V(P)$.

\subsection{The case of a bounded domain}

Until now we have considered our functionals as defined on subsets of the whole $\mathbb{R}^{2}$. For applications to image processing it is important to consider subsets of a fixed bounded domain. We briefly see how results in Theorems 3.14 and 3.6 can fit this case with some little modification.

Let $\Omega \subset \mathbb{R}^{2}$ be a bounded star-shaped open set with Lipschitz boundary and set:

$$
\begin{aligned}
& \mathcal{A}^{\Omega}:=\left\{E \subset \Omega: \partial E \cap \Omega \text { is } C^{2}\right\} \\
& \mathcal{R}^{\Omega}:=\{P \subset \Omega: \exists \tilde{P} \in \mathcal{R} \text { such that } \tilde{P} \cap \Omega=P\} \\
& \mathcal{P}^{\Omega}:=\{P \subset \Omega: \exists \tilde{P} \in \mathcal{P} \text { such that } \tilde{P} \cap \Omega=P\} .
\end{aligned}
$$

For the sake of simplicity we can suppose that $\Omega$ is star-shaped with respect to the origin.

Denote by $V(P)$ the set of the vertices of $\partial P$ contained in $\Omega$. Remark that, if $\partial P \cap \partial \Omega$ is not empty, we do not consider as elements of $V(P)$ the end-points of each connected component of $\partial P \cap \Omega$.

We call efficient boundary of $P$ the following set: $\tilde{\partial} P:=\overline{\partial P \backslash \partial \Omega}$ and we denote by $\tilde{V}(P)$ the set of the vertices of $\tilde{\partial} P$. In general $\tilde{V}(P)$ is greater or equal than $V(P)$. In Figure 3 we picture an example describing the difference between $V(P)$ and $\tilde{V}(P)$.

Analogously to the definitions in (3.2), (3.4) and (3.5), we denote by

$$
\mathcal{G}_{\varepsilon}: \mathcal{A}^{\Omega} \rightarrow[0,+\infty], \quad \mathcal{G}_{\mathcal{R}}: \mathcal{P}^{\Omega} \rightarrow[0,+\infty] \text { and } \mathcal{G}: \mathcal{P}^{\Omega} \rightarrow[0,+\infty]
$$

the following functionals:

$$
\begin{aligned}
\mathcal{G}_{\varepsilon}(E) & =\int_{\partial E \cap \Omega}\left(\frac{\varphi(x, \tau)}{\varepsilon}+\varepsilon \kappa^{2}(x)\right) \mathrm{d} \mathcal{H}^{1}(x) \\
\mathcal{G}_{\mathcal{R}^{\Omega}}(P) & = \begin{cases}\sum_{p \in V(P) \backslash\left\{p_{1}, \ldots, p_{M}\right\}} g\left(p, v^{-}(p), v^{+}(p)\right) & \text { if } P \in \mathcal{R}^{\Omega} \\
+\infty & \text { otherwise }\end{cases} \\
\mathcal{G}(P) & =\inf \left\{\liminf _{n \rightarrow+\infty} \mathcal{G}_{\mathcal{R}^{\Omega}}\left(P_{n}\right): P_{n} \rightarrow P \text { in } L^{1}(\Omega)\right\},
\end{aligned}
$$

where $g$ is defined as in (3.3). 
For the family $\left\{\mathcal{G}_{\varepsilon}\right\}$ it can be proved a result of compactness with respect to the $L^{1}(\Omega)$-topology and the proof essentially follows the same strategy in that of Theorem 3.14.

Moreover the family $\left\{\mathcal{G}_{\varepsilon}\right\} \Gamma$-converges to $\mathcal{G}$ with respect to the $L^{1}(\Omega)$-topology. We do not include the detailed proof in this case, just pointing out the main differences with the case treated in Theorem 3.6. First of all, in the case of a bounded domain, the boundaries of the approximating sets and of the limit sets are union of not necessarily closed curves. However the several definitions in the lemmata used to prove the main results can be rephrased in this framework. Another difference is that the functional $\mathcal{G}$ does not weigh the vertices on the boundary of $\Omega$. In this case for the $\Gamma$-lim inf inequality we can reason as in the proof of Theorem 3.6 and we note that the lower estimate improves when we remove the terms (in the $\Gamma$-limit) related to the vertices on the boundary. To recover the optimal sequence for the $\Gamma$-limsup inequality we reason as follows. If $P$ is a polygon such that $\bar{P} \subset \Omega$ (and hence $\partial P \cap \partial \Omega=\emptyset$ ) or if $\partial P \cap \partial \Omega \neq \emptyset$ and $V(P) \equiv \tilde{V}(P)$, then we consider the sequence $\left\{E_{\varepsilon}\right\}$ given by Theorem 3.6 (for the case when the ambient space is $\mathbb{R}^{2}$ ) and we can take as optimal sequence the family $\left\{E_{\varepsilon} \cap \Omega\right\}$. If instead $V(P)$ is strictly contained in $\tilde{V}(P)$, then we consider the dilation $T^{\eta}: \mathbb{R}^{2} \rightarrow \mathbb{R}^{2}$, defined for $\eta>0$ as: $T^{\eta}(x)=\eta x$. For $\eta$ small enough we define $P^{\eta}:=T^{\eta}(P)$ and we choose $\eta$ in such a way that $V\left(P^{\eta}\right) \equiv \tilde{V}\left(P^{\eta}\right)$. In general $P^{\eta}$ could not belong to $\mathcal{P}^{\Omega}$ : this happen if there are segments of the boundary of $P$ pointing to a focal point. In this case however, for every $\varepsilon_{\eta}>0$, we can take a polygon $\tilde{P}^{\eta} \in \mathcal{P}^{\Omega}$ such that $\left\|\chi_{\tilde{P}^{\eta}}-\chi_{P^{\eta}}\right\|_{L^{1}(\Omega)}<\varepsilon_{\eta}$. Now, since $\tilde{P}^{\eta}$ belongs to $\mathcal{P}^{\Omega}$, we can extend it to a polygon $\bar{P}^{\eta}$ belonging to $\mathcal{P}$. Let $\left\{E_{\varepsilon}^{\eta}\right\}$ be the optimal sequence given by Theorem 3.6 for $\bar{P}^{\eta}$ and let us consider the family of sets $\left\{E_{\varepsilon}^{\eta} \cap \Omega\right\}$. By a diagonal argument we recover an optimal approximating sequence for $P$ in such a way that $\mathcal{G}$ does not weigh the vertices of $P$ lying on the boundary of $\Omega$.

Remark 3.18. If $\Omega$ is a bounded domain and all the focal points belong to $\mathbb{R}^{2} \backslash \bar{\Omega}$, we can slightly modify the definition of $G$ considering the following functional:

$$
F(P):=\inf \left\{\liminf _{n \rightarrow+\infty} G_{\mathcal{R}}\left(P_{n}\right): P_{n} \rightarrow P \text { in } L^{1}(\Omega), \sup _{n} \mathcal{H}^{1}\left(\partial P_{n}\right)<+\infty\right\},
$$

which in this case is lower semicontinuous with respect to the $L^{1}(\Omega)$-topology. We have that the family $\left\{G_{\varepsilon}\right\}$ $\Gamma$-converges to $F$ with respect to the $L^{1}(\Omega)$-topology with uniform bounds of the perimeters, that means:

- for every $P \in \mathcal{P}$ and every sequence $\left\{E_{n}\right\} \subset \mathcal{A}$ (with $\sup _{n} \mathcal{H}^{1}\left(\partial E_{n}\right)<+\infty$ ) converging to $P \Rightarrow$ $G(P) \leq \liminf _{n \rightarrow+\infty} G_{\varepsilon_{n}}\left(E_{n}\right)$

- for every $P \in \mathcal{P}$ there exists a sequence $\left\{E_{n}\right\} \subset \mathcal{A}$ (with $\left.\sup _{n} \mathcal{H}^{1}\left(\partial E_{n}\right)<+\infty\right)$ converging to $P$ such that $G(P) \geq \limsup _{n \rightarrow+\infty} G_{\varepsilon_{n}}\left(E_{n}\right)$.

By Theorem 2.4 and Proposition 2.5 we deduce that, up to a subsequence, the solutions of problem

$$
\min \left\{\int_{\partial E}\left(\frac{\varphi(x, \tau(x))}{\varepsilon}+\varepsilon \kappa^{2}(x)\right) \mathrm{d} \mathcal{H}^{1}(x)+\int_{\mathbb{R}^{2}}\left|\chi_{E}-\chi_{A}\right|^{2} \mathrm{~d} x\right\}
$$

converge to solutions of problem

$$
\min _{P \in \mathcal{P}}\left\{F(P)+\int_{\mathbb{R}^{2}}\left|\chi_{P}-\chi_{A}\right|^{2} \mathrm{~d} x\right\}
$$

\section{Appendix}

\subsection{Proof of Lemma 2.10}

We perform the construction for a single $B_{\delta}\left(p_{j}\right)$, which can be iterated for each $j=1, \ldots, M$ (we can suppose that $\partial B_{\delta}\left(p_{i}\right) \cap \partial B_{\delta}\left(p_{j}\right)=\emptyset$ for $\left.i \neq j\right)$. Set $\mathcal{U}:=\partial B_{\delta}\left(p_{j}\right) \cap \partial P$. By assumption on $P$ we have that the number of points of $\mathcal{U}$ is finite and we set $\mathcal{U}:=\left\{q_{1}, \ldots, q_{l}\right\}$. Moreover, without loss of generality, we can assume that $V(P) \cap \partial B_{\delta}\left(p_{j}\right)=\emptyset$ and then $l$ is even, because $\partial P$ is closed. Let us consider the points $\left\{q_{1}, \ldots, q_{l}\right\}$ ordered 


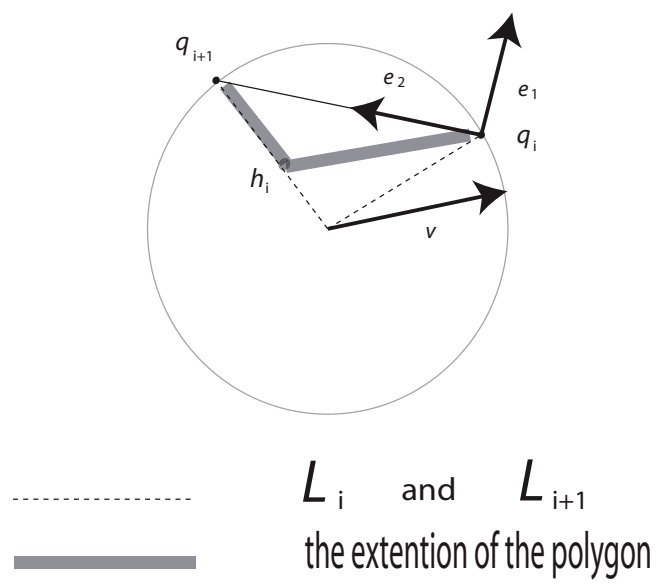

Figure 4. A possible extension of $P$ in $B_{\delta}\left(p_{j}\right)$ when there exists at least a vector $v \in$ $\left\{\tau_{1}, \ldots, \tau_{N}\right\}$ such that $\delta v+p_{j} C_{\delta}\left(q_{1}, q_{2}\right) \cup C_{\delta}\left(-q_{2},-q_{1}\right)$.

in the natural way induced by the identification of $B_{\delta}\left(p_{j}\right)$ with $\mathbb{R}$ modulo $2 \pi$. With a little abuse of notation we denote this ordering by the usual symbol " $<$ " and for points $y_{1}, y_{2} \in \partial B_{\delta}\left(p_{j}\right)$ with $y_{1}<y_{2}$ we define $C_{\delta}\left(y_{1}, y_{2}\right)$ as the closed arc connecting $y_{1}$ and $y_{2}$ in $\partial B_{\delta}\left(p_{j}\right)$. For $x \in \partial B_{\delta}\left(p_{j}\right)$ let $\left.\nu_{(} x\right)$ be the outer normal unit vector and define $\psi(x)=\tau(x) \cdot \nu(x)$. By the geometrical properties stated before for $P$, it is easy to see that $\psi\left(q_{i}\right) \cdot \psi\left(q_{i+1}\right)<0$. Let us now modify $P$ in $B_{\delta}\left(p_{j}\right)$. For each $q_{i}$, with $i$ odd, consider the segments $L_{i}:=\left[q_{i}, p_{j}\right]$ and $L_{i+1}:=\left[q_{i+1}, p_{j}\right]$ and distinguish the two following cases.

Case 1. There exists at least a vector $v \in\left\{\tau_{1}, \ldots, \tau_{N}\right\}$ such that $\delta v+p_{j} \notin C_{\delta}\left(q_{1}, q_{2}\right) \cup C_{\delta}\left(-q_{2},-q_{1}\right)$. In this case let $e_{1}$ and $e_{2}$ be the two orthogonal unit vectors defined as:

$$
e_{2}:=\frac{q_{1}+q_{2}-2 p_{j}}{\left|q_{1}+q_{2}-2 p_{j}\right|}, \quad e_{1}:=-e_{2}^{\perp} .
$$

Now if

$$
\frac{v \cdot e_{2}}{v \cdot e_{1}}<0
$$

then let $r_{i}$ be the straight line passing thought $q_{i}$ and parallel to $v$ and set $h_{i}:=L_{i+1} \cap r_{i}$ (condition (4.1) ensures that such $h_{i}$ exists). The required extension of $P$ in $B_{\delta}\left(p_{j}\right)$ will be the union of the two segments $\left[q_{i}, h_{i}\right]$ and $\left[h_{i}, q_{i+1}\right]$ (see Fig. 4$)$.

Moreover, if $\psi\left(q_{i}\right)$ is negative, we will take a parameterization of $\left[q_{i}, h_{i}\right] \cup\left[h_{i}, q_{i+1}\right]$ in such a way to go from $q_{i}$ to $q_{i+1}$, while in the case $\psi\left(q_{i}\right)>0$ we consider the path going from $q_{i+1}$ to $q_{i}$.

If instead

$$
\frac{v \cdot e_{2}}{v \cdot e_{1}}>0
$$

we can repeat the previous steps but taking

$$
h_{i}=L_{i} \cap r_{i+1},
$$

where $r_{i+1}$ is the straight line passing though $q_{i+1}$ and parallel to $v$. 


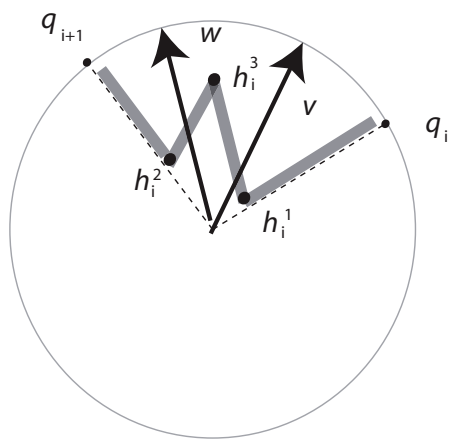

Figure 5. A possible extension of $P$ in $B_{\delta}\left(p_{j}\right)$ when all the fixed directions $\tau_{1}, \ldots, \tau_{N}$ belong to $C_{\delta}\left(q_{i}, q_{i+1}\right) \cup C_{\delta}\left(-q_{i+1},-q_{i}\right)$.

Case 2. All the fixed directions $\tau_{1}, \ldots, \tau_{N}$ belong to $C_{\delta}\left(q_{i}, q_{i+1}\right) \cup C_{\delta}\left(-q_{i+1},-q_{i}\right)$. Let $v, w \in\left\{ \pm \tau_{1}, \ldots, \pm \tau_{N}\right\}$ such that:

$$
v \cdot w>0 \quad \text { and } \quad \frac{v \cdot e_{2}}{v \cdot e_{1}}<\frac{w \cdot e_{2}}{w \cdot e_{1}} .
$$

For $h_{i}^{1} \in\left[q_{i}, p_{j}\right]$ and $h_{i}^{2} \in\left[q_{i+1}, p_{j}\right]$ let $L_{i}$ be the straight line passing through $h_{i}^{1}$ and parallel to $w$ and $L_{i+1}$ be the straight line passing through $h_{i}^{2}$ and parallel to $v$. We chose $h_{i}^{1}$ and $h_{i}^{2}$ in such a way that $h_{i}^{3}=L_{i} \cap L_{i+1}$ belongs to $B_{\delta}\left(p_{j}\right)$. The required extension of $P$ in $B_{\delta}\left(p_{j}\right)$ in this case is obtained taking the set $\left[q_{i}, h_{i}^{1}\right] \cup\left[h_{i}^{1}, h_{i}^{3}\right] \cup\left[h_{i}^{3}, h_{i}^{2}\right] \cup\left[h_{i}^{2}, q_{i+1}\right]$, paying attention also in this case to the versus of the parameterization according to the sign of $\psi\left(q_{i}\right)$ (see Fig. 5).

\subsection{Proof of Lemma 3.5}

By the regularity of $\varphi$ we can write

$$
\int_{a}^{b}\left(\frac{\varphi(\alpha(s), \dot{\alpha}(s))}{\varepsilon}+\varepsilon|\ddot{\alpha}(s)|^{2}\right) \mathrm{d} s=\int_{a}^{b}\left(\frac{\varphi(q, \dot{\alpha}(s))}{\varepsilon}+\varepsilon|\ddot{\alpha}(s)|^{2}\right) \mathrm{d} s+\int_{a}^{b} \frac{\nabla_{x} \varphi(\zeta, \dot{\alpha}(s)) \cdot(\alpha(s)-q)}{\varepsilon} \mathrm{d} s
$$

for suitable $\zeta \in(\alpha(s), q)$. For the first term in the sum in (4.3) we have:

$$
\begin{aligned}
\int_{a}^{b}\left(\frac{\varphi(q, \dot{\alpha}(s))}{\varepsilon}+\varepsilon|\ddot{\alpha}(s)|^{2}\right) \mathrm{d} s & \geq 2 \int_{a}^{b} \sqrt{\varphi(q, \beta(s))}|\dot{\beta}(s)| \mathrm{d} s \\
& \geq 2 \int_{C\left(\nu_{1}+\eta, \nu_{2}-\eta\right)} \sqrt{\varphi(q, \xi)} \mathrm{d} \mathcal{H}^{1}(\xi) \\
& \geq 2 \int_{C\left(\nu_{1}, \nu_{2}\right)} \sqrt{\varphi(q, \xi)} \mathrm{d} \mathcal{H}^{1}(\xi)+o_{\eta}(1)=g\left(q, \nu_{1}, \nu_{2}\right)+o_{\eta}(1)
\end{aligned}
$$

where we have set $\beta(s):=\dot{\alpha}(s)$. For the second term in the sum in (4.3) we have:

$$
\int_{a}^{b} \frac{\nabla_{x} \varphi(\zeta, \dot{\alpha}(s)) \cdot(\alpha(s)-q)}{\varepsilon} \mathrm{d} s=o_{\varepsilon}(1)
$$

In fact by the definition of $\varphi$ in (3.1) it follows that there exists $c_{1}, c_{2}>0$ such that:

$$
\left|\nabla_{x} \varphi(x, \tau)\right| \leq c_{1} \rho_{\varepsilon}+c_{2} \rho_{\varepsilon}^{2} \quad \forall x \in B_{\rho_{\varepsilon}}, \forall \tau \in S^{1} .
$$


By (4.6) and (3.34) we get:

$$
\left|\int_{a}^{b} \frac{\nabla_{x} \varphi(\zeta, \dot{\alpha}(s)) \cdot(\alpha(s)-q)}{\varepsilon} \mathrm{d} s\right| \leq \frac{1}{\varepsilon} \int_{a}^{b}\left|\nabla_{x} \varphi(\zeta, \dot{\alpha}(s))\right| \cdot|(\alpha(s)-q)| \mathrm{d} s \leq 2 M_{1} \frac{\rho_{\varepsilon}^{2}}{\varepsilon}\left(c_{1}+c_{2} \rho_{\varepsilon}\right),
$$

which is the thesis, choosing $\rho_{\varepsilon}=o(\sqrt{\varepsilon})$.

\section{REFERENCES}

[1] L. Ambrosio and A Braides, Functionals defined on partitions of sets of finite perimeter, I and II. J. Math. Pures. Appl. 69 (1990) 285-305 and 307-333.

[2] L. Ambrosio and V.M. Tortorelli, Approximation of functionals depending on jumps by elliptic functionals via $\Gamma$-convergence. Comm. Pure Appl. Math. 43 (1990) 999-1036.

[3] L. Ambrosio, N. Fusco and D. Pallara, Functions of Bounded Variation and Free Discontinuity Problems. Clarendon Press, Oxford (2000).

[4] G. Aubert and P. Kornprobst, Mathematical problems in image processing. Partial differential equations and the calculus of variations. Springer, New York (2006).

[5] G. Bellettini and R. March, An image segmentation variational model with free discontinuities and contour curvature. Math. Mod. Meth. Appl. Sci. 14 (2004) 1-45.

[6] G. Bellettini and L. Mugnai, Characterization and representation of the lower semicontinuous envelope of the elastica functional. Ann. Inst. Henri Poincaré, Anal. Non Linéaire 21 (2004) 839-880.

[7] G. Bellettini, G. Dal Maso and M. Paolini, Semicontinuity and relaxation properties of a curvature depending functional in 2D. Ann. Scuola Norm. Sup. Pisa (4) 20 (1993) 247-297.

[8] A. Blake and A. Zisserman, Visual Reconstruction. MIT Press, Cambridge, MA (1987).

[9] A. Braides, Approximation of Free-Discontinuity Problems, Lecture Notes in Mathematics. Springer-Verlag, Berlin (1998).

[10] A. Braides, Г-Convergence for Beginners. Oxford University Press, Oxford (2002).

[11] A. Braides and A. Malchiodi, Curvature theory of boundary phases: the two-dimensional case. Interfaces Free Bound. 4 (2002) 345-370.

[12] A. Braides and R. March, Approximation by $\Gamma$-convergence of a curvature-depending functional in visual reconstruction. Comm. Pure Appl. Math. 59 (2006) 71-121.

[13] A. Braides, A. Chambolle and M. Solci, A relaxation result for energies defined on pairs set-function and applications. ESAIM: COCV 13 (2007) 717-734.

[14] A. Chambolle, Image segmentation by variational methods: Mumford and Shah functional and the discrete approximations. SIAM J. Appl. Math. 55 (1995) 827-863.

[15] A. Chambolle, Finite-differences discretizations of the Mumford-Shah functional. ESAIM: M2AN 33 (1999) 261-288.

[16] A. Chambolle and G. Dal Maso, Discrete approximation of the Mumford-Shah functional in dimension two. ESAIM: M2AN 33 (1999) 651-672.

[17] A. Coscia, On curvature sensitive image segmentation. Nonlin. Anal. 39 (2000) 711-730.

[18] G. Dal Maso, An Introduction to $\Gamma$-Convergence. Birkhäuser, Boston (1993).

[19] G. Dal Maso, J.M. Morel and S. Solimini, A variational method in image segmentation: existence and approximation results. Acta Math. 168 (1992) 89-151.

[20] C. Mantegazza, Curvature varifolds with boundary. J. Diff. Geom. 43 (1996) 807-843.

[21] R. March, Visual reconstruction with discontinuities using variational methods. Image Vis. Comput. 10 (1992) 30-38.

$[22]$ L. Modica and S. Mortola, Il limite nella $\Gamma$-convergenza di una famiglia di funzionali ellittici. Boll. Un. Mat. Ital. A (5) 3 (1977) $526-529$.

[23] J.M. Morel and S. Solimimi, Variational Methods in Image Segmentation, Progress in Nonlinear Differential Equations and Their Applications 14. Birkhäuser, Basel (1995).

[24] D. Mumford, Elastica and computer vision, in Algebraic Geometry and its Applications (West Lafayette, IN 1990), Springer, New York (1994) 491-506.

[25] D. Mumford and J. Shah, Optimal approximations by piecewise smooth functions and associated variational problems. Comm. Pure Appl. Math. 42 (1989) 577-685.

[26] M. Nitzberg, D. Mumford and T. Shiota, Filtering, Segmentation and Depth, in Lecture Notes in Computer Science 662, Springer-Verlag, Berlin (1993).

[27] M. Röger and R. Schätzle, On a modified conjecture of De Giorgi. Math. Z. 254 (2006) 675-714.

[28] J. Shah, Uses of elliptic approximations in computer vision, in Variational Methods for Discontinuous Structures, Birkhäuser, Basel (1996) 19-34.

[29] J. Shah, A common framework for curve evolution, segmentation and anisotropic diffusion, in IEEE Conference on Computer Vision and Pattern Recognition, June (1996). 